\title{
GLOBAL PROPAGATION ON CAUSAL MANIFOLDS ${ }^{\ddagger}$
}

\author{
ANDREA D'AGNOLO§ AND PIERRE SCHAPIRA ${ }^{\Uparrow}$
}

1. Introduction. The micro-support of sheaves (see [7]) is a tool to describe local propagation results. A natural problem is then to give sufficient conditions to get global propagation results from the knowledge of the micro-support. This is the aim of this paper.

A propagator on a real manifold $M$ is the data of a pair $(Z, \lambda)$, where $Z \subset M \times M$ is a closed subset containing the diagonal, $\lambda$ is a closed cone of the cotangent bundle to $M$, and some relation holds between $\lambda$ and the micro-support of the constant sheaf along $Z$. In this framework, we prove that if $F$ is a sheaf on $M$ whose microsupport does not intersect $-\lambda$ outside of the zero-section, then the restriction morphism $\mathrm{R} \Gamma(M ; F) \rightarrow \mathrm{R} \Gamma(U ; F)$ is an isomorphism, as soon as $M \backslash U$ is $Z$-proper. This last condition means that the forward set $D^{\uparrow}=\{y \in M:(x, y) \in Z$ for some $x \in D\}$ of any compact set $D \subset M$ should intersect $M \backslash U$ in a compact set, and the backward set $(M \backslash U)^{\downarrow}=\{x \in M:(x, y) \in Z$ for some $y \notin U\}$ should not contain any connected component of $M$.

As an application, we consider the problem of global existence for solutions to hyperbolic systems (in the hyperfunction and distribution case), along the lines of Leray [8]. Causal manifolds, and in particular homogeneous causal manifolds as considered by Faraut et al., give examples of manifolds to which our results apply.

\section{Statement of the results.}

2.1. Normal cones. A subset $C$ of a finite dimensional real vector space $V$ is called a cone (or a conic subset), if $\mathbb{R}^{+} \cdot C \subset C$. A cone $C \subset V$ is called convex if $C+C \subset C$, and proper if $\bar{C} \cap-\bar{C} \subset\{0\}$. We also use the notation $C^{a}=-C$. Denoting by $V^{*}$ the dual of $V$, the polar to a cone $C \subset V$ is the conic subset of $V^{*}$ defined by $C^{\circ}=\{\xi:\langle\xi, v\rangle \geq 0$ for every $v \in C\}$. One checks that $\left(C^{\circ}\right)^{\circ}$ is the closure of the convex envelop to $C$, and that the polar to a proper convex cone is a closed proper convex cone.

Let $M$ be a $C^{\infty}$-manifold. If $q: E \rightarrow M$ is a vector bundle, one naturally extends the above notions to subsets of $E$. For example, $\gamma \subset E$ is a cone if $\gamma_{x}:=\gamma \cap q^{-1}(x)$ is a cone in $E_{x}$ for any $x \in X$. We identify $M$ to the zero-section of $q$, and for $\gamma \subset E$ we set $\dot{\gamma}=\gamma \backslash M$.

Denote by $\tau: T M \rightarrow M$ and $\pi: T^{*} M \rightarrow M$ the tangent and cotangent bundle to $M$, respectively. Following [7, Definition 4.1.1], $C(A, B)$ denotes the Whitney normal cone of $A, B \subset M$, a closed cone of $T M$. Recall that if $(x)$ is a local coordinate system in $M$, then $\left(x_{\circ} ; v_{\circ}\right) \in C(A, B)$ if and only if there exists a sequence $\left(a_{n}, b_{n}, c_{n}\right)$ in $A \times B \times \mathbb{R}^{+}$such that

$$
a_{n} \rightarrow x_{\circ}, \quad b_{n} \rightarrow x_{\circ}, \quad c_{n}\left(a_{n}-b_{n}\right) \rightarrow v_{\circ} .
$$

If $N \subset M$ is a smooth submanifold, $C_{N}(A)$ is the projection of $C(N, A)$ in $T_{N} M$, the normal bundle to $N$ in $M$.

\footnotetext{
${ }^{\ddagger}$ Received November 20, 1998; accepted for publication December 28, 1998.

$\S$ Institute of Mathematics, University Pierre et Marie Curie, Case 82, 4 Place Jussieu, F-75252 Paris Cedex 05, France (dagnolo@math.jussieu.fr).

I Institute of Mathematics, University Pierre et Marie Curie, Case 82, 4 Place Jussieu, F-75252 Paris Cedex 05, France (schapira@math.jussieu.fr).
} 
The strict normal cone to $A \subset M$ is defined in [7, Definition 5.3.6] by $N(A)=$ $T M \backslash C(M \backslash A, A)$. Recall that if $(x)$ is a local coordinate system in $M$, then $\left(x_{\circ} ; v_{\circ}\right) \in$ $N(A)$ if and only if there exists an open cone $C$ containing $v_{\circ}$, and a neighborhood $U$ of $x_{\circ}$, such that

$$
U \cap((A \cap U)+C) \subset A
$$

Note that $N(A)$ is an open convex cone of $T M, N(M \backslash A)=N(A)^{a}$, and $N_{x}(A) \neq$ $T_{x} M$ if and only if $x$ is in the topological boundary of $A$.

2.2. Micro-support. Let $M$ be a $C^{\infty}$-manifold. Let $k$ be a field, and denote by $\mathbf{D}^{\mathrm{b}}\left(k_{M}\right)$ the bounded derived category of sheaves of $k$-vector spaces on $M$. Following [7, Chapter 5], to $F \in \mathbf{D}^{\mathrm{b}}\left(k_{M}\right)$ one associates its micro-support $S S(F)$, a closed conic involutive subset of $T^{*} M$. Recall that $T^{*} M \backslash S S(F)$ describes the (co)directions of propagation for the cohomology of $F$, stable by small perturbations. More precisely, $p \notin S S(F)$ if and only if there exists an open neighborhood $\Omega$ of $p$ such that for any $x \in \pi(\Omega)$ and any $C^{\infty}$-function $\varphi$ on $M$ with $\varphi(x)=0, d \varphi(x) \in \Omega$, one has

$$
\left(\mathrm{R} \Gamma_{\{\varphi \geq 0\}} F\right)_{x}=0,
$$

where $\mathrm{R} \Gamma_{W}$ denotes the derived functor of sections with support on a closed subset $W \subset M$, and we write for short $\{\varphi \geq 0\}=\{y \in M: \varphi(y) \geq 0\}$. This is indeed a propagation requirement, since the above vanishing can be restated by asking that the natural restriction morphism

$$
\underset{U \ni x}{\lim _{U}} H^{j}(U ; F) \rightarrow \underset{U \ni x}{\lim _{U}} H^{j}(U \cap\{\varphi<0\} ; F)
$$

is an isomorphism for any $j \in \mathbb{Z}$. This implies that "sections" of $F$ on $U \cap\{\varphi<0\}$ extend to a neighborhood of $x$.

If $A \subset M$ is a locally closed subset, denote by $k_{A}$ the sheaf on $M$ which is zero on $M \backslash A$, and constant with fiber $k$ on $A$. Recall that if $U \subset M$ is an open subset, and $W \subset M$ is a closed subset, one has the estimates:

$$
S S\left(k_{U}\right) \subset N(U)^{\circ a}, \quad S S\left(k_{W}\right) \subset N(W)^{\circ} .
$$

2.3. Propagators. Let $M$ be a $C^{\infty}$-manifold. Denote by $\Delta \subset M \times M$ the diagonal, and by $q_{1}$ and $q_{2}$ the first and second projection from $M \times M$ to $M$.

Definition 2.1. Let $Z \subset M \times M$ be a closed subset. We say that a locally closed subset $A \subset M$ is $Z$-proper if

(i) $q_{1}$ is proper on $Z \cap q_{2}^{-1}(\bar{A})$,

(ii) $q_{1}\left(Z \cap q_{2}^{-1}(\bar{A})\right)$ does not contain any connected component of $M$.

Given $Z \subset M \times M$ as above, to a subset $A \subset M$, we associate

$$
\begin{aligned}
& A^{\downarrow}=q_{1}\left(Z \cap q_{2}^{-1} A\right), \\
& A^{\uparrow}=q_{2}\left(Z \cap q_{1}^{-1} A\right),
\end{aligned}
$$

and we set $x^{\downarrow}=\{x\}^{\downarrow}, x^{\uparrow}=\{x\}^{\uparrow}$. With these notations, a subset $A \subset M$ is $Z$-proper if and only if: (i) $D^{\uparrow} \cap \bar{A}$ is compact for any compact subset $D$ of $M$, (ii) $\bar{A}^{\downarrow}$ does not contain any connected component of $M$.

Definition 2.2. Let $Z$ be a closed subset of $M \times M$, and $\lambda$ a closed cone of $T^{*} M$. We say that the pair $(Z, \lambda)$ is a propagator on $M$ if 
(2.4) $\Delta \subset Z$,

(2.5) $S S\left(k_{Z}\right) \subset T^{*} M \times \lambda$,

(2.6) $S S\left(k_{Z}\right) \cap\left(T^{*} M \times M\right) \subset M \times M$,

(2.7) $S S\left(k_{Z}\right) \cap\left(M \times T^{*} M\right) \subset M \times M$.

(As for (2.6) and (2.7), recall that we identify the zero-section of $T^{*} M$ to $M$.) We say that $(Z, \lambda)$ is a convex propagator on $M$ if it is a propagator and moreover

(2.8) $\lambda$ is a proper convex cone.

2.4. Propagation theorems. We can now state our main result.

Theorem 2.3. Let $(Z, \lambda)$ be a propagator on $M$. Let $F \in \mathbf{D}^{\mathrm{b}}\left(k_{M}\right)$, and assume that $\operatorname{supp}(F)$ is $Z$-proper and $S S(F) \cap \lambda^{a} \subset M$. Then

$$
\mathrm{R} \Gamma(M ; F)=0 .
$$

Part (i) of the following corollary partially extends to manifolds Proposition 5.2.1 of [7] which only considered an affine situation, with $\lambda$ constant along the fibers. (See Remark 2.6 for further comments.)

Corollary 2.4. Let $(Z, \lambda)$ be a convex propagator on $M$. Let $F \in \mathbf{D}^{\mathrm{b}}\left(k_{M}\right)$, and assume that $S S(F) \cap \lambda^{a} \subset M$.

(i) Let $W$ be a closed subset of $M$ which is Z-proper and satisfies $S S\left(k_{W}\right) \subset \lambda^{a}$. Then

$$
\mathrm{R} \Gamma_{W}(M ; F)=0 .
$$

(ii) Let $U$ be an open subset of $M$ which is Z-proper and satisfies $S S\left(k_{U}\right) \subset \lambda$. Then

$$
\mathrm{R} \Gamma\left(M ; F_{U}\right)=0 .
$$

Note that (i) and (ii) are equivalent to

$$
\begin{aligned}
& \mathrm{R} \Gamma(M ; F) \stackrel{\sim}{\longrightarrow} \mathrm{R} \Gamma(M \backslash W ; F), \\
& \mathrm{R} \Gamma(M ; F) \stackrel{\sim}{\rightarrow} \mathrm{R} \Gamma(M \backslash U ; F),
\end{aligned}
$$

respectively. In other words, "sections" of $F$ on $M \backslash W$ (or on a neighborhood of $M \backslash U)$ extend uniquely to $M$.

The following result deals with the case where $\lambda$ is not convex, but is covered by a finite union of convex cones. A situation which appears for example in dealing with the Cauchy problem, real or complex.

Corollary 2.5. Let $I$ be a finite set. For $j \in I$, let $U_{j}$ be an open subset of $M$, and set $N=M \backslash \bigcup_{j \in I} U_{j}$. For any $J \subset I, J \neq \varnothing$, let $\left(Z_{J}, \lambda_{J}\right)$ be a convex propagator, and set $U_{J}=\bigcap_{j \in J} U_{j}$. Let $F \in \mathrm{D}^{\mathrm{b}}\left(k_{M}\right)$. Assume

(i) $U_{J}$ is $Z_{J}$-proper, and $S S\left(k_{U_{J}}\right) \subset \lambda_{J}$,

(ii) $S S(F) \cap \lambda_{J}^{a} \subset M$.

Then, one has the isomorphism

$$
\mathrm{R} \Gamma(M ; F) \stackrel{\sim}{\longrightarrow} \mathrm{R} \Gamma(N ; F) .
$$


REMARK 2.6. Theorem 2.3 does not allow one to recover Proposition 5.2.1 of [7], since our hypotheses are stronger. More precisely, we require $\lambda$ closed proper convex and $S S(F) \cap \lambda^{a} \subset M$, while in loc. cit. one only assumes $\lambda=\gamma^{\circ}$, for $\gamma$ closed proper convex in $T M$, and $S S(F) \cap \operatorname{Int}\left(\lambda^{a}\right)=\varnothing$. Let us give an example which shows that, in general, it is not possible to replace the hypothesis $S S(F) \cap \lambda^{a} \subset M$ by the hypothesis $S S(F) \cap \operatorname{Int}\left(\lambda^{a}\right)=\varnothing$.

Let $M=\mathbb{R} \times S^{1}$ be an infinite cylinder. Using the identification $T^{*} M=M \times(\mathbb{R} \times$ $\mathbb{R})$, set $Z=\left\{\left(x_{1}, \theta_{1}, x_{2}, \theta_{2}\right) \in M \times M: x_{1}=x_{2}\right\}, \lambda=\left\{(x, \theta ; \xi, \tau) \in T^{*} M: \tau \geq 0\right\}$, $W=\{0\} \times S^{1} \subset M$. Clearly, $(Z, \lambda)$ is a propagator on $M$, and $W$ is $Z$-proper. Setting $F=k_{W}$, one has $S S(F)=\left\{(x, \theta ; \xi, \tau) \in T^{*} M: x=\tau=0\right\}$, and hence

$$
\begin{aligned}
& S S(F) \cap \operatorname{Int}\left(\lambda^{a}\right)=\varnothing, \\
& S S(F) \cap \lambda^{a} \not \subset M, \\
& \Gamma(M ; F) \neq 0 .
\end{aligned}
$$

\section{Proof of the results.}

3.1. Review on sheaves. Let $f: N \rightarrow M$ be a morphism of $C^{\infty}$ manifolds. We will consider the usual operations $R f_{*}, R f_{!}, f^{-1}, f^{!}, \otimes, R \mathcal{H}$ om of sheaf theory. If $F \in \mathbf{D}^{\mathrm{b}}\left(k_{M}\right)$, we set $D^{\prime} F=R \mathcal{H o m}\left(F, k_{M}\right)$. We also make use of the absolute and relative dualizing complexes denoted $\omega_{M}$ and $\omega_{N / M}$, respectively. Recall that if $f$ is smooth, then $\omega_{N / M}=\operatorname{or}_{N / M}[\operatorname{dim} N-\operatorname{dim} M]$, where or $N / M$ denotes the relative orientation sheaf.

We will need the following lemma.

LEMMA 3.1. Let $Z$ be a closed subset of $M \times M$ containing the diagonal $\Delta$. Then

(i) $k_{M}$ is a direct summand of $R q_{2 *} k_{Z}$,

(ii) $\omega_{M}$ is a direct summand of $R q_{2 !} \omega_{Z}$.

Proof. Since the arguments are similar, we will prove only (ii). Set $\widetilde{q}_{2}=\left.q_{2}\right|_{z}$, and denote by $i: \Delta \rightarrow Z$ the closed embedding. Note that $\widetilde{q}_{2} \circ i$ gives an identification $\Delta \simeq M$. Applying Verdier adjunction formula thrice, we get the commutative diagram

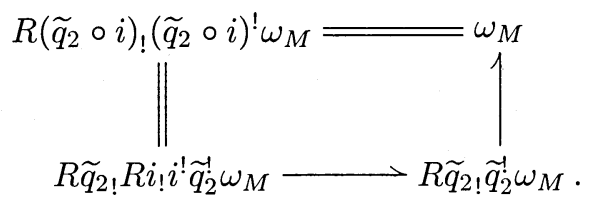

In other words, the identity of $\omega_{M}$ factorizes through $R \widetilde{q}_{2 !} \widetilde{q}_{2}^{4} \omega_{M} \simeq R q_{2 !} \omega_{Z}$. One concludes by using [7, Exercise 1.4].

Finally, let us list some functorial properties that the micro-support enjoys, referring to [7] for proofs.

Consider the correspondence of cotangent bundles associated to $f$ :

$$
T^{*} N \underset{{ }_{t} f^{\prime}}{\overleftarrow{\leftarrow}} N \times_{M} T^{*} M \underset{f_{\pi}}{\longrightarrow} T^{*} M
$$

Let $F \in \mathbf{D}^{\mathrm{b}}\left(k_{M}\right)$ and assume $f$ is smooth, then $f^{!} F \simeq \omega_{N / M} \otimes f^{-1} F$ and

$$
S S\left(f^{-1} F\right) \subset{ }^{t} f^{\prime} f_{\pi}^{-1}(S S(F)) .
$$


Let $G \in \mathbf{D}^{\mathrm{b}}\left(k_{N}\right)$ and assume $f$ is proper on $\operatorname{supp}(G)$, then $R f_{!} G \simeq R f_{*} G$ and

$$
S S\left(R f_{*} G\right) \subset f_{\pi}^{t} f^{\prime-1}(S S(G)) .
$$

Let $F, G \in \mathbf{D}^{\mathrm{b}}\left(k_{M}\right)$ and assume $S S(F) \cap S S(G)^{a} \subset M$, then

$$
S S(F \otimes G) \subset S S(F)+S S(G) .
$$

Let $F, G \in \mathbf{D}^{\mathrm{b}}\left(k_{M}\right)$ and assume $S S(F) \cap S S(G) \subset M$, then

$$
S S(R \mathcal{H o m}(G, F)) \subset S S(F)+S S(G)^{a} \text {. }
$$

3.2. Review on kernels. Consider the natural projections $M \underset{q_{1}}{\overleftarrow{ }} M \times M \underset{q_{2}}{\longrightarrow}$ $M$. To $K \in \mathbf{D}^{\mathrm{b}}\left(k_{M \times M}\right)$ one associates the functors

$$
\Phi_{K}(F)=R q_{2 !}\left(K \otimes q_{1}^{-1} F\right), \quad \Psi_{K}(F)=R q_{1 *} R \mathcal{H o m}\left(K, q_{2}^{!} F\right) .
$$

These two functors are adjoint to each other, i.e., for $F, G \in \mathbf{D}^{\mathrm{b}}\left(k_{M}\right)$

$$
\operatorname{RHom}\left(\Phi_{K}(F), G\right) \simeq \operatorname{RHom}\left(F, \Psi_{K}(G)\right) .
$$

Using the estimates we recalled in the previous section, one easily gets the following result.

Proposition 3.2. Let $F \in \mathbf{D}^{\mathrm{b}}\left(k_{M}\right)$ and $K \in \mathbf{D}^{\mathrm{b}}\left(k_{M \times M}\right)$.

(i) Assume that $q_{2}$ is proper on $\operatorname{supp}(K) \cap q_{1}^{-1} \operatorname{supp}(F)$ and that one has the estimate $S S(K)^{a} \cap(S S(F) \times M) \subset M \times M$. Then one has the estimate

$$
S S\left(\Phi_{K}(F)\right) \subset\left\{(y ; \eta):(x, y ; \xi, \eta) \in S S(K) \text { for some }(x ; \xi) \in S S(F)^{a}\right\} .
$$

(ii) Assume that $q_{1}$ is proper on $\operatorname{supp}(K) \cap q_{2}^{-1} \operatorname{supp}(F)$ and that one has the estimate $S S(K) \cap(M \times S S(F)) \subset M \times M$. Then one has the estimate

$$
S S\left(\Psi_{K}(F)\right) \subset\left\{(x ; \xi):(x, y ; \xi, \eta) \in S S(K)^{a} \text { for some }(y ; \eta) \in S S(F)^{a}\right\} .
$$

3.3. Proof of Theorem 2.3. Let us consider the kernel $K=R j_{\text {! }} \omega_{Z}$, where $j$ denotes the embedding $Z \subset M \times M$. Since $K \simeq R \mathcal{H o m}\left(k_{Z}, \omega_{M \times M}\right)$, by (2.8) one has $S S(K) \subset S S\left(k_{Z}\right)^{a}$. By (2.7) and the fact that $q_{1}$ is proper on $Z \cap q_{2}^{-1} W$, the hypotheses of Proposition 3.2 (ii) are satisfied. We find

$$
S S\left(\Psi_{K}(F)\right) \subset\left\{(x ; \xi):(x, y ; \xi, \eta) \in S S\left(k_{Z}\right) \text { for some }(y ; \eta) \in S S(F)^{a}\right\} .
$$

Let $(x, y ; \xi, \eta) \in S S\left(k_{Z}\right)$ with $(y ; \eta) \in S S(F)^{a}$. Hypothesis $(2.5)$ together with the fact that $S S(F) \cap \lambda^{a} \subset M$, imply that $(y ; \eta) \in M$, and then hypothesis (2.6) implies $(x ; \xi) \in M$. We thus have $S S\left(\Psi_{K}(F)\right) \subset M$, and hence $\Psi_{K}(F)$ is locally constant on $M$. On the other hand, one has the estimate $\operatorname{supp}\left(\Psi_{K}(F)\right) \subset q_{1}\left(Z \cap q_{2}^{-1} W\right)=W^{\downarrow}$, and $W^{\downarrow}$ does not contain any connected component of $M$ by hypothesis. Hence $\Psi_{K}(F)=0$.

By the same argument we obtain $\Psi_{K}\left(F \otimes \omega_{M}\right)=0$, and hence

$$
0=\operatorname{RHom}\left(k_{M}, \Psi_{K}\left(F \otimes \omega_{M}\right)\right) \simeq \operatorname{RHom}\left(\Phi_{K}\left(k_{M}\right), F \otimes \omega_{M}\right) .
$$

Since $\Phi_{K}\left(k_{M}\right) \simeq R q_{2 !} \omega_{Z}$, Lemma 3.1 (ii) implies

$$
0=\mathrm{RHom}\left(\omega_{M}, F \otimes \omega_{M}\right) \simeq \operatorname{RHom}\left(k_{M}, F\right) .
$$

REMARK 3.3. As it is clear from the above proof, one could generalize the notion of propagator by considering pairs $(K, \lambda)$, for $K \in \mathrm{D}^{\mathrm{b}}\left(k_{M \times M}\right)$. In this case, one should replace $Z$-proper by $\operatorname{supp}(K)$-proper, and hypothesis $(2.4)$ by the following requirement: there exist $G \in \mathbf{D}^{\mathrm{b}}\left(k_{M}\right)$ and a locally free sheaf of rank one $L$ on $M$, such that $L$ is a direct summand of $\Phi_{K}(G)$. 
3.4. Proof of Corollary 2.4. Let us prove (i). Since $S S(F) \cap S S\left(k_{W}\right) \subset M$, we get by (3.4) that $S S\left(\mathrm{R} \Gamma_{W} F\right) \subset S S(F)+\lambda$. Since $S S(F) \cap \lambda^{a} \subset M$ and $\lambda$ is a proper convex closed cone, this implies

$$
S S\left(\mathrm{R} \Gamma_{W} F\right) \cap \lambda^{a} \subset M .
$$

We may then apply Theorem 2.3 with $F$ replaced by $\mathrm{R} \Gamma_{W} F$.

The proof of (ii) is almost the same, noticing that since $S S(F) \cap S S\left(k_{U}\right)^{a} \subset M$, we get by (3.3) that $S S\left(F_{U}\right) \subset S S(F)+\lambda$.

3.5. Proof of Corollary 2.5. Applying the functor $\mathrm{R} \Gamma(M ; \cdot \otimes F)$ to the exact sequence

$$
0 \rightarrow k_{M \backslash N} \rightarrow k_{M} \rightarrow k_{N} \rightarrow 0
$$

we are reduced to prove

$$
\mathrm{R} \Gamma\left(M ; F_{M \backslash N}\right)=0 .
$$

By the hypotheses, one has the isomorphism in $\mathbf{D}^{\mathrm{b}}\left(k_{M}\right)$

$$
k_{M \backslash N} \simeq\left(0 \rightarrow k_{U_{I}} \rightarrow \cdots \rightarrow \bigoplus_{J \subset I,|J|=2} k_{U_{J}} \rightarrow \bigoplus_{i \in I} k_{U_{i}} \rightarrow 0\right)
$$

where $\bigoplus_{i \in I} k_{U_{i}}$ is in degree zero. Hence, it is enough to prove that

$$
\mathrm{R} \Gamma\left(M ; F_{U_{J}}\right)=0 \text { for any } J \subset I .
$$

This follows from Corollary 2.4 (ii).

4. Applications to hyperbolic systems. In this section, $M$ is a real analytic manifold, and $k=\mathbb{C}$.

4.1. Hyperfunction solutions. We refer to Sato [9, 10], Sato-Kawai-Kashiwara [11], and Kashiwara [5], for the notions of hyperfunction, wave-front set, and $\mathcal{D}$ module, that we shall use.

Let $X$ be a complexification of $M$. Following [7, $\S 6.2]$, using the natural projection $T_{M}^{*} X \rightarrow M$ and the Hamiltonian isomorphism, we will identify $T^{*} M$ to a subset of the normal bundle $T_{T_{M}^{*} X} T^{*} X$.

Let us denote by $\mathcal{O}_{X}$ and $\mathcal{D}_{X}$ the sheaves of holomorphic functions and of linear partial differential operators, respectively. If $\mathcal{M}$ is a coherent $\mathcal{D}_{X}$-module (i.e., a system of P.DE), we denote by $\operatorname{char}(\mathcal{M})$ its characteristic variety, a closed $\mathbb{C}^{\times}$-conic involutive subvariety of $T^{*} X$.

Definition 4.1. (cf [6]) Let $\lambda \subset T^{*} M$ be a closed cone, and $\mathcal{M}$ a coherent $\mathcal{D}_{X}$-module. One says that $\mathcal{M}$ is $\lambda$-hyperbolic if

$$
\lambda \cap C_{T_{M}^{*} X}(\operatorname{char}(\mathcal{M})) \subset M .
$$

(Note that, since $\operatorname{char}(\mathcal{M})$ is $\mathbb{C}^{\times}$-conic, $\mathcal{M}$ is $\lambda$-hyperbolic if and only if it is $\lambda^{a}$ hyperbolic.)

Recall that the sheaf $\mathcal{B}_{M}$ of Sato's hyperfunctions on $M$ is given by $\mathcal{B}_{M}:=$ $R \mathcal{H o m}\left(D^{\prime} k_{M}, \mathcal{O}_{X}\right) \simeq H_{M}^{\operatorname{dim} M}\left(\mathcal{O}_{X}\right) \otimes \operatorname{or}_{M / X}$. 
TheOREM 4.2. Let $(Z, \lambda)$ be a convex propagator on $M$, and $W \subset M$ a closed $Z$ proper subset satisfying $S S\left(k_{W}\right) \subset \lambda^{a}$. Let $\mathcal{M}$ be a coherent $\mathcal{D}_{X}$-module and assume it is $\lambda$-hyperbolic. Then

$$
\mathrm{R} \Gamma\left(M ; \operatorname{RHom}_{\mathcal{D}_{X}}\left(\mathcal{M}, \Gamma_{W} \mathcal{B}_{M}\right)\right)=0
$$

Proof. Setting $F=R \mathcal{H}$ om $_{\mathcal{D}_{X}}\left(\mathcal{M}, \mathcal{B}_{M}\right)$, it follows from [6] or [7, $\left.\S 11.5\right]$ (see also [1] for the case of a single operator) that $S S(F) \subset C_{T_{M}^{*} X}(\operatorname{char}(\mathcal{M}))$. Since $\mathcal{B}_{M}$ is a flabby sheaf, one has

$$
\mathrm{R} \Gamma\left(M ; \operatorname{RHom}_{\mathcal{D}_{X}}\left(\mathcal{M}, \Gamma_{W} \mathcal{B}_{M}\right)\right)=\mathrm{R}_{W}(M ; F) .
$$

The result then follows from Corollary 2.4 (i).

Let $N \subset M$ be a real analytic submanifold, and denote by $Y \subset X$ a complexification. One says that $Y$ is non-characteristic for a coherent $\mathcal{D}_{X}$-module $\mathcal{M}$, if

$$
T_{Y}^{*} X \cap \operatorname{char}(\mathcal{M}) \subset X
$$

In this case, the induced system $\mathcal{M}_{Y}$ is a coherent $\mathcal{D}_{Y}$-module. Note that if $\mathcal{M}$ is $T_{N}^{*} M$-hyperbolic, then $Y$ is non-characteristic for $\mathcal{M}$.

THEOREM 4.3. Let $N \subset M$ be a real analytic submanifold, and $I$ a finite set. For $j \in I$, let $U_{j}$ be open subsets of $M$, such that $N=M \backslash \bigcup_{j \in I} U_{j}$. For any $J \subset I$, $J \neq \varnothing$, let $\left(Z_{J}, \lambda_{J}\right)$ be a convex propagator, and set $U_{J}=\bigcap_{j \in J} U_{j}$. Assume that $U_{J}$ is $Z_{J}$-proper, and $S S\left(k_{U_{J}}\right) \subset \lambda_{J}$. Let $\mathcal{M}$ be a coherent $\mathcal{D}_{X}$-module and assume it is $\lambda_{J}$-hyperbolic for any $J \subset I$. Then, one has the isomorphism

$$
\mathrm{R} \Gamma\left(M ; \text { RHom }_{\mathcal{D}_{X}}\left(\mathcal{M}, \mathcal{B}_{M}\right)\right) \stackrel{\sim}{\longrightarrow} \mathrm{R} \Gamma\left(N ; R \mathcal{H o m}{ }_{\mathcal{D}_{Y}}\left(\mathcal{M}_{Y}, \mathcal{B}_{N}\right)\right)
$$

Note that the same statement holds when replacing Sato's hyperfunctions by real analytic functions.

Proof. Applying Corollary 2.5 with $F=R \mathcal{H}$ om $_{\mathcal{D}_{X}}\left(\mathcal{M}, \mathcal{B}_{M}\right)$, we get

$$
\mathrm{R} \Gamma\left(M ; \text { RHom }_{\mathcal{D}_{X}}\left(\mathcal{M}, \mathcal{B}_{M}\right)\right) \stackrel{\sim}{\longrightarrow} \mathrm{R} \Gamma\left(N ; \text { RHom }\left._{\mathcal{D}_{X}}\left(\mathcal{M}, \mathcal{B}_{M}\right)\right|_{N}\right) .
$$

It follows by (3.7) that $S S\left(k_{M \backslash N}\right) \subset \bigcup_{J} \lambda_{J}$. Since $T_{N}^{*} M$ coincides with $S S\left(k_{M \backslash N}\right)$ outside of the zero section, the fact that $\mathcal{M}$ is $\lambda_{J}$-hyperbolic for any $J \subset I$ implies that $\mathcal{M}$ is $T_{N}^{*} M$-hyperbolic. It then follows from [6] or [7, $\left.\S 11.5\right]$ that

$$
\left.R \mathcal{H o m}_{\mathcal{D}_{X}}\left(\mathcal{M}, \mathcal{B}_{M}\right)\right|_{N} \simeq R \mathcal{H} \operatorname{Hom}_{\mathcal{D}_{Y}}\left(\mathcal{M}_{Y}, \mathcal{B}_{N}\right)
$$

Let $P$ be a differential operator on $X$, and denote by $\sigma(P)$ its principal symbol, a homogeneous function on $T^{*} X$. One says that $P$ is $\lambda$-hyperbolic if so is the associated $\mathcal{D}$-module $\mathcal{M}=\mathcal{D}_{X} / \mathcal{D}_{X} P$. If $(z)=(x+i y)$ is a local coordinate system in $X$, and $(z ; \zeta)=(x+i y ; \xi+i \eta)$ the associated symplectic coordinates in $T^{*} X$, then $P$ is $\lambda$-hyperbolic if and only if

$$
\sigma(P)(x ; i \eta+\theta) \neq 0 \quad \text { for any }(x ; i \eta) \in T_{M}^{*} X,(x ; \theta) \in \lambda, \theta \neq 0 .
$$


Corollary 4.4. Let $(Z, \lambda)$ be a convex propagator on $M$, and $W \subset M$ a closed $Z$-proper subset satisfying $S S\left(k_{W}\right) \subset \lambda^{a}$. Let $P$ be a differential operator on $X$ and assume it is $\lambda$-hyperbolic. Then $P$ induces an isomorphism

$$
P: \Gamma_{W}\left(M ; \mathcal{B}_{M}\right) \stackrel{\sim}{\rightarrow} \Gamma_{W}\left(M ; \mathcal{B}_{M}\right)
$$

Proof. Apply Theorem 4.2 with $\mathcal{M}=\mathcal{D}_{X} / \mathcal{D}_{X} P$, and note that the solution complex $R \mathcal{H}$ om $_{\mathcal{D}_{X}}\left(\mathcal{M}, \Gamma_{W} \mathcal{B}_{M}\right)$ is represented by the complex of flabby sheaves

$$
0 \rightarrow \Gamma_{W} \mathcal{B}_{M} \underset{P}{\rightarrow} \Gamma_{W} \mathcal{B}_{M} \rightarrow 0
$$

Denote by $r$ the automorphism of $M \times M$ given by $r(x, y)=(y, x)$.

Corollary 4.5. Let $N \subset M$ be a real analytic hypersurface dividing $M$ in two closed half-spaces $N^{ \pm}$, and let $\theta$ be an analytic vector field defined in a neighborhood of $N$ and normal to it. Let $(Z, \lambda)$ be a convex propagator on $M$, and assume that $N^{+}$ is $Z$-proper, $N^{-}$is $r(Z)$-proper, and $S S\left(k_{N^{+}}\right) \subset \lambda^{a}$. Let $P$ be a differential operator on $X$, and assume it is $\lambda$-hyperbolic. Then $P$ induces a surjective morphism

$$
P: \Gamma\left(M ; \mathcal{B}_{M}\right) \rightarrow \Gamma\left(M ; \mathcal{B}_{M}\right),
$$

and moreover the homogeneous Cauchy problem

$$
\left\{\begin{array}{l}
P u=0, \\
\gamma_{\theta}(u)=\left(w_{1}, \ldots, w_{m}\right),
\end{array}\right.
$$

is globally well posed in the framework of hyperfunctions. (Here, $m$ is the order of $P$, and the trace map $\gamma_{\theta}(u)=\left(\left.u\right|_{N},\left.\theta u\right|_{N}, \ldots,\left.\theta^{m-1} u\right|_{N}\right)$ is well defined since $P u=0$ implies that the wave-front of $u$ is transversal to $N$.)

4.2. Distribution solutions. As above, let $X$ be a complexification of $M$. We denote by $\mathcal{D} b_{M}$ the sheaf of Schwartz distributions on $M$.

DeFinition 4.6. Let $\mathcal{M}$ be a coherent $\mathcal{D}_{X}$-module.

(i) We say that $\mathcal{M}$ is $\mathcal{D} b$-hyperbolic at $p \in T^{*} M$ if

$$
p \notin S S\left(R \mathcal{H} o m_{\mathcal{D}_{X}}\left(\mathcal{M}, \mathcal{D} b_{M}\right)\right) .
$$

(ii) Let $\lambda \subset T^{*} M$ be a closed cone. One says that $\mathcal{M}$ is $\lambda$-D $b$-hyperbolic if it is $\mathcal{D} b$-hyperbolic at any $p \in \lambda \backslash M$, i.e. if

$$
\lambda \cap S S\left(R \mathcal{H} \operatorname{Hom}_{\mathcal{D}_{X}}\left(\mathcal{M}, \mathcal{D} b_{M}\right)\right) \subset M .
$$

With this definition, it is clear that Corollary 2.4 (i) implies

THEOREM 4.7. Let $(Z, \lambda)$ be a convex propagator on $M$, and $W \subset M$ a closed $Z$ proper subset satisfying $S S\left(k_{W}\right) \subset \lambda^{a}$. Let $\mathcal{M}$ be a coherent $\mathcal{D}_{X}$-module, and assume it is $\lambda$-D b-hyperbolic. Then

$$
\mathrm{R} \Gamma\left(M ; \operatorname{RHom}_{\mathcal{D}_{X}}\left(\mathcal{M}, \mathrm{R} \Gamma_{W} \mathcal{D} b_{M}\right)\right)=0 .
$$

REMARK 4.8. The problem, of course, is to give conditions for a system $\mathcal{M}$ to be $\mathcal{D} b$-hyperbolic. If $P$ is a differential operator on $X$, and $\mathcal{M}=\mathcal{D}_{X} / \mathcal{D}_{X} P$, then it 
is well-known that $\mathcal{M}$ is $\mathcal{D} b$-hyperbolic if: it is hyperbolic, has characteristics with real constant multiplicities, and it satisfies the Levi conditions. An analog statement holds for systems (not necessarily determined) by [2]. Little is known beside the case of real constant multiplicities, or of constant coefficients in $\mathbb{R}^{n}$.

Let us now consider the case of a single differential operator $P$. One says that $P$ is $\mathcal{D} b$-hyperbolic at $p$ (resp. $\lambda$-D $b$-hyperbolic) if so is the system $\mathcal{M}=\mathcal{D}_{X} / \mathcal{D}_{X} P$.

Corollary 4.9. Let $(Z, \lambda)$ be a convex propagator on $M$, and $W \subset M$ a closed $Z$-proper subset satisfying $S S\left(k_{W}\right) \subset \lambda^{a}$. Let $P$ be a $\lambda$-DD-hyperbolic differential operator on $X$, and assume that $P: \mathcal{D} b_{M} \rightarrow \mathcal{D} b_{M}$ is stalk-wise surjective. Then $P$ induces isomorphisms

$$
\begin{aligned}
& P: \Gamma_{W}\left(M ; \mathcal{D} b_{M}\right) \stackrel{\sim}{\rightarrow} \Gamma_{W}\left(M ; \mathcal{D} b_{M}\right), \\
& P: H_{W}^{1}\left(M ; \mathcal{D} b_{M}\right) \stackrel{\sim}{\longrightarrow} H_{W}^{1}\left(M ; \mathcal{D} b_{M}\right) .
\end{aligned}
$$

Proof. Set $\mathcal{D} b_{M}^{P}=\operatorname{ker}\left(P: \mathcal{D} b_{M} \rightarrow \mathcal{D} b_{M}\right)$. Since $P: \mathcal{D} b_{M} \rightarrow \mathcal{D} b_{M}$ is an epimorphism, we have an isomorphism $R \mathcal{H}$ om $_{\mathcal{D}_{X}}\left(\mathcal{D}_{X} / \mathcal{D}_{X} P, \mathcal{D} b_{M}\right) \simeq \mathcal{D} b_{M}^{P}$, and a short exact sequence

$$
0 \rightarrow \mathcal{D} b_{M}^{P} \rightarrow \mathcal{D} b_{M} \underset{P}{\rightarrow} \mathcal{D} b_{M} \rightarrow 0
$$

Applying the functor $\mathrm{R} \Gamma_{W}(M, \cdot)$, we get the long exact cohomology sequence

$$
\begin{aligned}
0 & \rightarrow \Gamma_{W}\left(M ; \mathcal{D} b_{M}^{P}\right) \rightarrow \Gamma_{W}\left(M ; \mathcal{D} b_{M}\right) \underset{P}{\rightarrow} \Gamma_{W}\left(M ; \mathcal{D} b_{M}\right) \\
& \rightarrow H_{W}^{1}\left(M ; \mathcal{D} b_{M}^{P}\right) \rightarrow H_{W}^{1}\left(M ; \mathcal{D} b_{M}\right) \underset{P}{\rightarrow} H_{W}^{1}\left(M ; \mathcal{D} b_{M}\right) \\
& \rightarrow H_{W}^{2}\left(M ; \mathcal{D} b_{M}^{P}\right) \rightarrow 0
\end{aligned}
$$

Theorem 4.7 implies $H_{W}^{j}\left(M ; \mathcal{D} b_{M}^{P}\right)=0$ for any $j$, and the proof is complete.

Let us discuss a sufficient condition for $P$ to be $\mathcal{D} b$-hyperbolic.

Proposition 4.10. Let $P$ be a differential operator on $X$, and let $p \in \dot{T}^{*} M$. Assume

(i) $\sigma(P)(p) \neq 0$

(ii) $P:\left(\mathcal{D} b_{M}\right)_{x} \rightarrow\left(\mathcal{D} b_{M}\right)_{x}$ is surjective for any $x$ in a neighborhood of $\pi(p)$,

(iii) there exists an open neighborhood $\Omega \subset T^{*} M$ of $p$ such that for any $x \in \pi(\Omega)$, and any $C^{\infty}$-function $\varphi$ on $M$ with $\varphi(x)=0, d \varphi(x) \in \Omega$, one has

(iii) $)_{1}$ given $u \in\left(\Gamma_{\{\varphi<0\}} \mathcal{D} b_{M}\right)_{x}$ satisfying $P u=0$, there exists $\tilde{u} \in\left(\mathcal{D} b_{M}\right)_{x}$ such that $\left.\tilde{u}\right|_{\{\varphi<0\}}=u$ and $P \tilde{u}=0$,

(iii) $)_{2}$ given $v \in\left(\Gamma_{\{\varphi<0\}} \mathcal{D} b_{M}\right)_{x}$ there exists $u \in\left(\Gamma_{\{\varphi<0\}} \mathcal{D} b_{M}\right)_{x}$ such that $P u=v$.

Then, $P$ is $\mathcal{D} b$-hyperbolic at $p$.

Note that in (i) we used the embedding $T^{*} M \hookrightarrow M \times M T^{*} X$, which exists since $X$ is a complexification of $M$.

Proof. Since conditions (i)-(iii) are open in $p \in T^{*} M$, we may find an open neighborhood $\Omega$ of $p$ in $T^{*} M$ such that (iii) holds, (ii) holds in $\pi(\Omega)$, and moreover $\sigma(P)(q) \neq 0$ for any $q \in \Omega$. Let $x \in \pi(\Omega)$, and $\varphi$ be a $C^{\infty}$-function on $M$ as in (iii).

Consider the morphism of exact sequences, where the vertical arrows are induced 
by $P$

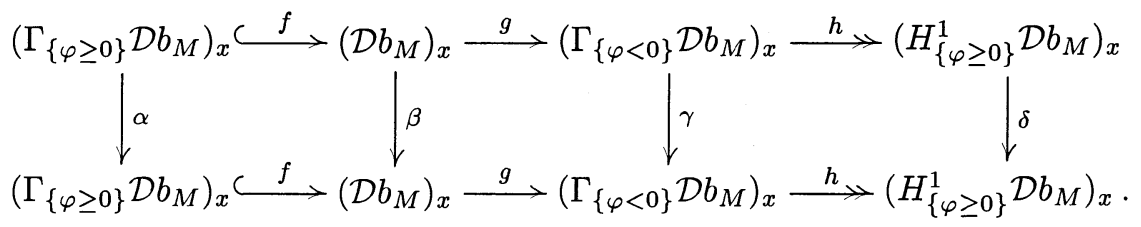

Consider the stalk-wise analog of (4.1) for $W=\{\varphi \geq 0\}$. By definition of the microsupport, we are left to prove that $\alpha$ and $\delta$ are isomorphisms. This follows from the following considerations. Hypothesis (i) states that $\{\varphi=0\} \subset M$ is non-characteristic for $P$, and by Holmgren's theorem this implies that $\alpha$ is injective. By (ii), $\beta$ is surjective. Moreover, hypothesis (iii) $)_{2}$ says that $\gamma$ is surjective, while hypothesis (iii) $)_{1}$ reads $g: \operatorname{ker} \beta \rightarrow \operatorname{ker} \gamma$.

REMARK 4.11. In his fundamental paper [8], Jean Leray discusses, among other topics, the problem of global extension for solutions to hyperbolic operators with simple characteristics. In particular, in loc. cit. it is shown that such operators satisfy the hypotheses of Proposition 4.10.

\section{Causal manifolds.}

5.1. Conal manifolds. In this section, we shall construct convex propagators.

DEFINITION 5.1. We say that a cone $\gamma \subset T M$ is admissible if it is closed proper convex and $\operatorname{Int}\left(\gamma_{x}\right) \neq \varnothing$ for any $x \in M$. (Here, $\operatorname{Int}\left(\gamma_{x}\right)$ denotes the interior of $\left.\gamma_{x}.\right)$ If $\gamma \subset T M$ is an admissible cone, we say that a closed subset $Z \subset M \times M$ is a $\gamma$-propagator if

(5.1) $\Delta \subset Z$,

(5.2) $N(Z) \supset(M \times \operatorname{Int}(\gamma)) \cup\left(\operatorname{Int}(\gamma)^{a} \times M\right)$.

(As for (5.2), recall that we identify the zero-section of $T M$ to $M$. .)

Proposition 5.2. If $\gamma \subset T M$ is an admissible cone and $Z \subset M \times M$ is a $\gamma$-propagator, then $\left(Z, \gamma^{\circ}\right)$ is a convex propagator.

Proof. If $\gamma \subset T M$ is admissible, then $\lambda=\gamma^{\circ}$ satisfies (2.8).

If $V_{1}$ and $V_{2}$ are two real finite dimensional vector spaces, we identify $\left(V_{1} \times V_{2}\right)^{*}$ to $V_{1}^{*} \times V_{2}^{*}$ by $\left\langle\left(v_{1}, v_{2}\right),\left(\nu_{1}, \nu_{2}\right)\right\rangle=\left\langle v_{1}, \nu_{1}\right\rangle+\left\langle v_{2}, \nu_{2}\right\rangle$. Then, if $C_{1}$ and $C_{2}$ are two cones with $C_{1} \neq \varnothing, C_{2} \neq \varnothing$, one has $\left(C_{1} \times C_{2}\right)^{\circ}=C_{1}^{\circ} \times C_{2}^{\circ}$. In particular, since $\operatorname{Int}\left(\gamma_{x}\right) \neq \varnothing$ for any $x \in M$, one has $(M \times \operatorname{Int}(\gamma))^{\circ}=T^{*} M \times \gamma^{\circ}$. This last set contains $N(Z)^{\circ}$ by hypothesis (5.2). Using the estimate $(2.3),(2.5)$ follows.

Remark that if $C$ is an open convex cone in $V_{1} \times V_{2}$ and $\left(0, v_{2}\right) \in C$ for $v_{2} \neq 0$, then $C^{\circ} \cap\left(V_{1}^{*} \times\{0\}\right)=\{0\}$. By hypothesis (5.2), for each $x_{\circ}, y_{\circ} \in M$ there exists $0 \neq w_{\circ} \in T_{y_{\circ}} M$ with $\left(0, w_{\circ}\right) \in N(Z)_{\left(x_{\circ}, y_{\circ}\right)}$. Then $N(Z)^{\circ} \cap\left(T_{x_{\circ}}^{*} M \times\left\{y_{\circ}\right\}\right) \subset\{0\}$, and (2.6) follows.

The proof of (2.7) is similar.

Definition 5.3. A conal manifold is a $C^{\infty}$-manifold $M$ endowed with an admissible cone $\gamma \subset T M$. On a conal manifold $M$, a continuous piecewise smooth curve $\alpha:[0,1] \rightarrow M$ is called a $\gamma$-path if the derivative from the right $\alpha_{r}^{\prime}(t)$ exists for any $t \in\left[0,1\left[\right.\right.$, and moreover $\alpha_{r}^{\prime}(t) \in \gamma_{\alpha(t)}$. For $x, y \in M$, we write $x \preccurlyeq y$ if there exists a $\gamma$-path $\alpha:[0,1] \rightarrow M$ with $\alpha(0)=x, \alpha(1)=y$.

Clearly, $\preccurlyeq$ is a preorder relation. In general, the graph of $\preccurlyeq$ in $M \times M$ is not closed, and we consider its closure

$$
Z_{\gamma}=\overline{\{(x, y): x \preccurlyeq y\}} .
$$


Note that $Z_{\gamma}$ may fail to be the graph of a preordering, since transitivity may not hold.

Proposition 5.4. Let $\gamma \subset T M$ be an admissible cone. Then

(i) if $W \subset M$ is a closed subset with $W^{\downarrow}=W$, then $S S\left(k_{W}\right) \subset \gamma^{\circ a}$.

(ii) $Z_{\gamma}$ is a $\gamma$-propagator,

Proof. (i) By (2.3), it is enough to show that $N(W) \supset \operatorname{Int}\left(\gamma^{a}\right)$. Let $x_{\circ} \in W$, and $-v_{\circ} \in \operatorname{Int}\left(\gamma_{x_{\circ}}\right)$. There exist a local chart $U$ at $x_{\circ}$, and an open conic neighborhood $C$ of $v_{\circ}$ in $T_{x_{\circ}} M$, such that $U \times C \subset \gamma$. In view of (2.1), we shall prove that

$$
U \cap((W \cap U)-C) \subset W .
$$

Since $W=W^{\downarrow}$, if $\alpha$ is a $\gamma$-path and $\alpha(1) \in W$, then $\alpha(0) \in W$. Let $x \in W \cap U$ and $v \in C$ with $x-v \in U$. Since the segment of straight line from $x-v$ to $x$ is a $\gamma$-path, $x-v \in W$.

(ii) Let us prove that $N\left(Z_{\gamma}\right) \supset M \times \operatorname{Int}(\gamma)$. Let $\left(x_{\circ}, y_{\circ}\right) \in Z_{\gamma}$, and $w_{\circ} \in \operatorname{Int}\left(\gamma_{y_{\circ}}\right)$. Take a local chart $V$ at $y_{\circ}$ and an open conic neighborhood $C$ of $w_{\circ}$ in $T_{y_{\circ}} M$, such that $V \times C \subset \gamma$. Let $U \subset M$ be an open neighborhood of $x_{\circ}$. By (2.1), for any $x \in U$, $y \in V$, and $w \in C$, with $x \in y^{\downarrow}$, and $y+w \in V$, we have to show that $x \in(y+w)^{\downarrow}$. By definition, $x \in y^{\downarrow}$ if and only if there exist sequences $x_{n} \rightarrow x, y_{n} \rightarrow y$, with $x_{n} \preccurlyeq y_{n}$ (i.e., there is a $\gamma$-path from $x_{n}$ to $y_{n}$ ). We may assume $x_{n} \in U, y_{n}, y_{n}+w \in V$. Since $w \in C$, the segment of straight line from $y_{n}$ to $y_{n}+w$ is a $\gamma$-path. Composing the $\gamma$-paths above, we get $x_{n} \preccurlyeq y_{n}+w$, which implies $x \in(y+w)^{\downarrow}$ as requested.

The proof that $N\left(Z_{\gamma}\right) \supset \operatorname{Int}\left(\gamma^{a}\right) \times M$ is similar.

5.2. Causal manifolds. Recall that we denote by $\Delta \subset M \times M$ the diagonal, and by $q_{1}$ and $q_{2}$ the first and second projection from $M \times M$ to $M$. Moreover, for $i, j \in\{1,2,3\}$ let us denote by $q_{i j}$ the projection from $M \times M \times M$ to the corresponding factor $M \times M$ (e.g., $\left.q_{13}(x, y, z)=(x, z)\right)$. Recall that a preordering $\leq$ on $M$ is determined by its graph $Z=\{(x, y): x \leq y\}$, which is a subset of $M \times M$ satisfying

(5.4) $\Delta \subset Z$ (reflexivity),

(5.5) $q_{12}^{-1} Z \cap q_{23}^{-1} Z \subset q_{13}^{-1} Z$ (transitivity).

One says that $Z$ is a proper preordering if it is a preordering satisfying

(5.6) $Z \subset M \times M$ is closed, and $q_{13}$ is proper on $q_{12}^{-1} Z \cap q_{23}^{-1} Z$.

Note that the last condition in (5.6) means that $D^{\uparrow} \cap E^{\downarrow}$ is compact for any compact subsets $D$ and $E$ of $X$. In particular, this implies that the intervals $x^{\uparrow} \cap y^{\downarrow}$ are compact.

Definition 5.5. A causal manifold $M$ is the data of a manifold $M$ and of an admissible cone $\gamma \subset T M$ such that the set $Z_{\gamma}$ in (5.3) is a preordering. If moreover (5.3) is a proper preordering, $M$ is called a properly causal manifold.

Corollary 5.6. Let $M$ be a properly causal manifold, and $W$ a compact subset of $M$. If $W^{\downarrow}$ does not contain any connected component of $M$, then $W^{\downarrow}$ is $Z_{\gamma}$-proper and satisfies $S S\left(k_{W \downarrow}\right) \subset \gamma^{\circ a}$.

In other words, we are in a position to apply Corollary 2.4 .

Proof. Hypothesis (5.5) implies that $\left(W^{\downarrow}\right)^{\downarrow}=W^{\downarrow} \downarrow$. Hypothesis (5.6) implies that $D^{\uparrow} \cap W^{\downarrow}$ is compact for any compact subset $D$ of $M$. Finally, $S S\left(k_{W^{\downarrow}}\right) \subset \gamma^{\circ a}$ by Proposition 5.4 (ii).

5.3. Causal homogeneous spaces. The toy model for admissible cones is the one considered in [7], where $M$ is an open subset of a vector space $V$, and $\gamma=M \times C \subset$ $T M \simeq M \times V$ for a closed proper convex cone $C \subset V$. In other words, $\gamma$ is a constant cone field. In this case, using the notations of section 5.1, $x \preccurlyeq y$ reads $x-y \in C$, and 
$Z_{\gamma}=\{(x, y): x-y \in C\}$. This picture is invariant under the group of translations in $V$.

Less trivial examples are obtained by considering other Lie groups. Let $M=G / H$ be a homogeneous manifold, where $G$ is a real Lie group, and $H \subset G$ a closed subgroup. An admissible cone $\gamma \subset T M$ is called invariant if $\tau_{g}^{\prime}(x)\left(\gamma_{x}\right)=\gamma_{y}$ for $y=\tau_{g}(x)$, where $\tau_{g}$ denotes the $G$-action on $M, \tau_{g}(\tilde{g} H)=g \tilde{g} H$. One easily proves (see e.g. $[4, \S 2.2]$ )

PROPOSITION 5.7. If $\gamma \subset T M$ is an invariant admissible cone, then $Z_{\gamma}$ is the graph of a preordering.

Let us denote by $\leq$ the preordering defined by $Z_{\gamma}$. Clearly, this preordering is an invariant ordering, in the sense that for any $g \in G$, one has $\tau_{g}(x) \leq \tau_{g}(y)$ whenever $x \leq y$.

Denote by $\mathfrak{g}$ and $\mathfrak{h}$ the Lie algebras of $G$ and $H$, respectively. Denote by $e \in M$ the equivalence class of the identity element of $G$. Noticing that $T_{e} M=\mathfrak{g} / \mathfrak{h}$, it is clear that the data of an invariant admissible cone $\gamma \subset T M$ is equivalent to the data of a closed convex cone $C \subset \mathfrak{g}$ which is invariant by the adjoint action of $H$, and satisfies $C \cap C^{a}=\mathfrak{h}, C+C^{a}=\mathfrak{g}$.

Definition 5.8. A causal homogeneous space $M=G / H$ is the data of a real Lie group $G$, a closed subgroup $H \subset G$, and a cone $C \subset \mathfrak{g}$ satisfying the above properties. $M$ is called a properly causal homogeneous space if the associated causal manifold is properly causal.

If $(G, H)$ is a symmetric pair, refer to [4] for a wide family of examples of triples $(G, H, C)$ inducing a properly causal homogeneous structure on $M=G / H$.

Let us discuss a possible application of our results.

In [3], Faraut constructs global fundamental solutions to invariant hyperbolic differential operators in the framework of distributions. His method relies on the theory of constant coefficient hyperbolic operators and the technique of spherical transforms. Let us show how our results imply the existence of global fundamental solutions in the framework of hyperfunctions.

Assume that $(G, H, C)$ induces a properly causal homogeneous structure on $M=$ $G / H$. Let $P$ be an invariant differential operator on $M$ such that

$$
\sigma(P)(e ; i \eta+\theta) \neq 0 \quad \text { for any } \eta \in \mathfrak{g}^{*}, \theta \in C^{\circ}, \theta \neq 0 .
$$

If $e^{\downarrow}$ does not contains the connected component of $e$, we may apply Corollary 4.4 for $W=e^{\downarrow}$, and get the existence of a fundamental solution

$$
P u=\delta_{e}, \quad u \in \Gamma_{e^{\downarrow}}\left(M, \mathcal{B}_{M}\right) .
$$

\section{REFERENCES}

[1] Jean-Michel Bony and Pierre Schapira, Solutions hyperfonctions du problème de Cauchy, Lecture Notes in Mathematics, no. 287, Springer-Verlag, 1973, Proceedings Katata 1971, pp. 82-98.

[2] Andrea D'Agnolo and Francesco Tonin, Cauchy problem for hyperbolic D-modules with regular singularities, Pacific J. Math., 184:1 (1998), pp. 1-22.

[3] JACQUES FARAUT, Opérateurs différentiels invariants hyperboliques sur un espace symétrique ordonné, J. Lie Theory, 6:2 (1996), pp. 271-289.

[4] Joachim Hilgert and Gestur Ólafsson, Causal symmetric spaces, Perspectives in Mathematics, vol. 18, Academic Press Inc., San Diego, CA, 1997, Geometry and harmonic analysis.

[5] MASAKI KASHIWARA, Algebraic study of systems of partial differential equations (a translation by A. D'Agnolo and J.-P. Schneiders of Kashiwara's Master's Thesis, Tokyo University, December 1970), Mém. Soc. Math. France (N.S.), 63 (1995), xiv+72. 
[6] Masaki Kashiwara And Pierre Schapira, Micro-hyperbolic systems, Acta Math., 142 (1979), pp. 1-55.

[7] - Sheaves on manifolds, Grundlehren der Mathematischen Wissenschaften, 292, SpringerVerlag, Berlin, 1990.

[8] Jean Leray, Hyperbolic differential equations, The Institute for Advanced Study, Princeton, N. J., 1955.

[9] Miкio Sato, Theory of hyperfunctions. I, J. Fac. Sci. Univ. Tokyo. Sect. I, 8 (1959), pp. 139-193.

[10] —, Theory of hyperfunctions, II, J. Fac. Sci. Univ. Tokyo. Sect. I, 8 (1960), pp. 387-437.

[11] Mikio Sato, Takahiro Kawai, and Masaki Kashiwara, Microfunctions and pseudodifferential equations, Hyperfunctions and pseudo-differential equations, Lecture Notes in Math., Vol. 287, Springer, Berlin, 1973, pp. 265-529. 
